Nuclear Physics B205 [FS5] (1982) 239-252

(C) North-Holland Publishing Company

\title{
FINITE SIZE EFFECTS IN EUCLIDEAN LATTICE THERMODYNAMICS FOR NON-INTERACTING BOSE AND FERMI SYSTEMS
}

\author{
J. ENGELS; F. KARSCH and H. SATZ \\ Fakultät für Physik, Universität Bielefeld, Germany
}

Received 23 November 1981

\begin{abstract}
In the Monte Carlo simulation of QCD, the euclidean form of the partition function is evaluated on a finite lattice. We use this method to calculate the partition function for noninteracting Bose and Fermi fields. Here the expressions on the lattice can be evaluated in closed form and the continuum limit is well-known; this provides us with a measure for finite lattice size effects in such approaches.
\end{abstract}

\section{Introduction}

During the past year, the Monte Carlo simulation of Yang-Mills fields on a finite lattice has proven itself an extremely useful tool in the study of finite temperature thermodynamics for QCD systems. It is so far the only approach which allows a unified treatment over the entire temperature range, from the ideal gas at high temperature [1] through the deconfinement transition [1-3] into the non-perturbative phase [4]. It was noted in these studies, as well as in corresponding ones for the confinement problem, where the Monte Carlo approach to QCD was first introduced [5], that already for rather small lattices the results become roughly independent of lattice size. At $T=0$, it was moreover found that the lattice size dependence of the plaquette average is in accord with finite size scaling [6]. At finite temperatures, however, little is known of the effect that the finite lattice has on the results. It may be expected that the extension of the Monte Carlo methods to systems including fermions will for feasibility reasons require generally even smaller lattices [7], so any such effects will be even stronger here.

It therefore seems to us useful to investigate the effect of finite lattice structure by treating a system of non-interacting Bose and Fermi fields in the same euclidean lattice approach as used in QCD. We shall see that in these cases the partition function can be calculated in closed form on a finite lattice of any size. By comparing the results thus obtained with the well-known continuum forms for ideal Bose and Fermi gases, we can obtain a measure of finite lattice effects as well as some idea of what lattice structure provides the best approximation. 
The partition function for a system characterized by a lagrangian density $\mathscr{L}(\varphi)$, given in terms of Bose or Fermi fields $\varphi(x, t)$ and their derivatives, can be written as a functional integral [8]

$$
Z(\beta)=N(\beta) \int[\mathrm{d} \varphi] \exp \{-S(\varphi)\} ;
$$

the physical temperature $T=\beta^{-1}$ enters as boundary in the euclidean action,

$$
S(\varphi)=-\int_{0}^{\beta} \mathrm{d} \tau \int \mathrm{d}^{3} x \mathscr{L}(\varphi)
$$

with periodicity (antiperiodicity) for Bose (Fermi) fields

$$
\varphi_{\mathrm{B} / \mathrm{F}}(\boldsymbol{x}, 0)= \pm \varphi_{\mathrm{B} / \mathrm{F}}(\boldsymbol{x}, \boldsymbol{\beta}) \text {. }
$$

The normalization factor $N(\beta)$ is needed to assure the correct vacuum structure, since the functional integral alone still includes the contributions of the zero point $(T=0)$ terms.

In sect. 2 we evaluate $Z(\beta)$ on a finite $x-\tau$ lattice for non-interacting Bose fields, in sect. 3 for Fermi fields. In sect. 4, we then summarize the most important finite lattice features and their implications for general lattice calculations of thermodynamic systems.

\section{The ideal Bose gas on the euclidean lattice}

Free scalar Bose fields provide the simplest possible case of a finite temperature field theory, which can be solved exactly even in the continuum [8]. Here we want to put this theory on a euclidean lattice, in order to study the influence of a lattice cut-off on the thermodynamic quantities and to have a reference system for more complicated theories of interacting Bosons, like the pure gauge field part of QCD. There at present the lattice regularization seems to be the only way to get nonperturbative results for physical quantities.

Our aim is to calculate the partition function

$$
Z(\beta)=\operatorname{Tr} \mathrm{e}^{-\beta H},
$$

where the hamiltonian is given by

$$
H=\int \mathrm{d}^{3} x \mathscr{H}(\pi(x), \varphi(x))
$$

and $\mathscr{H}(\pi, \varphi)$ is the hamiltonian density of a free Bose field $\varphi$ and its conjugate momentum $\pi$ :

$$
\mathscr{H}(\pi, \varphi)=\frac{1}{2}\left(\pi^{2}+(\nabla \varphi)^{2}+m^{2} \varphi^{2}\right),
$$

$m$ is the mass of the boson. 
After integrating out the $\pi$-fields in the path-integral for $Z$ we get the euclidean version [eq. (1.1)] of eq. (2.1) with the euclidean action [8]

$$
S(\varphi)=\frac{1}{2} \int_{0}^{\beta} \mathrm{d} \tau \int \mathrm{d}^{3} x\left\{\left(\frac{\partial \varphi}{\partial \tau}\right)^{2}+(\nabla \varphi)^{2}+m^{2} \varphi^{2}\right\}
$$

On a finite lattice with $N_{\beta} \times N_{\sigma}^{3}$ sites and lattice spacings $a_{\beta}$ and $a_{\sigma}$ in the temperature and space directions $x$ has the values $x_{\alpha}=\left(\alpha_{0} a_{\beta}, \alpha a_{\sigma}\right)$, with integer $\alpha_{0}$ and $\alpha$. The partition function becomes

$$
Z_{\mathrm{E}}\left(N_{\sigma}, N_{\beta}, a_{\sigma}, a_{\beta}\right)=N^{\prime} \int \prod_{\alpha} \mathrm{d} \varphi\left(x_{\alpha}\right) \mathrm{e}^{-S(\varphi)},
$$

with

$$
\begin{gathered}
N^{\prime}=\left[\frac{a_{\sigma}^{3}}{2 \pi a_{\beta}}\right]^{N_{\sigma}^{3} N_{\beta} / 2} \\
S(\varphi)=\frac{1}{2} a_{\sigma}^{3} a_{\beta} \sum_{\alpha}\left\{\sum_{\mu=1}^{3}\left(\frac{\varphi\left(x_{\alpha}+\mathrm{e}_{\mu}\right)-\varphi\left(x_{\alpha}\right)}{a_{\sigma}}\right)^{2}+\left(\frac{\varphi\left(x_{\alpha}+\mathrm{e}_{0}\right)-\varphi\left(x_{\alpha}\right)}{a_{\beta}}\right)^{2}+m^{2} \varphi^{2}\left(x_{\alpha}\right)\right\} .
\end{gathered}
$$

Here $e_{\mu}, \mu=0,1,2,3$ are the lattice unit vectors and $\varphi\left(x_{\alpha}\right) \in \mathbb{R}$ are continuous site variables. We use periodic boundary conditions (pbc) in the space directions and in this section, for Bose field variables, we also require pbc in the $\beta$-direction. We have denoted the partition function as $Z_{\mathrm{E}}$, since it still has to be corrected for the $T=0$ contribution to agree with $Z=\operatorname{Tr} \exp (-\beta H)$. An additional problem arises for massless bosons. In this case the integral (2.5) as it stands is infinite, because the integrand is constant along the line of constant field configurations, $\varphi(x)=\varphi\left(x^{\prime}\right)$ for all sites, so that the integration along this line diverges. For the moment we therefore consider only $m \neq 0$; we shall show later how the results have to be corrected in the case $m=0$, which, of course, is of particular interest to us.

With the transformation

$$
\begin{gathered}
\tilde{\varphi}\left(x_{\alpha}\right) \equiv a_{\sigma} k^{-1 / 2} \varphi\left(x_{\alpha}\right), \\
k^{-1} \equiv 3 \xi^{-1}+\xi+\left(m a_{\sigma}\right)^{2} / 2 \xi, \\
\xi \equiv a_{\sigma} / a_{\beta},
\end{gathered}
$$

we introduce dimensionless variables. We then obtain from eq. (2.5)

$$
Z_{\mathrm{E}}\left(N_{\sigma}, N_{\beta}, a_{\sigma}, \xi\right)=\tilde{N}^{\prime} \int \prod_{\alpha} \mathrm{d} \tilde{\varphi}\left(x_{\alpha}\right) \mathrm{e}^{-S(\tilde{\varphi})},
$$

where

$$
\tilde{N}^{\prime}=[k \xi / 2 \pi]^{N_{3}^{3} N_{B} / 2}
$$


the quantity

$$
S=-\sum_{\alpha}\left\{-\tilde{\varphi}^{2}\left(x_{\alpha}\right)+K_{\sigma} \sum_{\mu=1}^{3} \tilde{\varphi}\left(x_{\alpha}+e_{\mu}\right) \tilde{\varphi}\left(x_{\alpha}\right)+K_{\beta} \tilde{\varphi}\left(x_{\alpha}+\mathrm{e}_{0}\right) \tilde{\varphi}\left(x_{\alpha}\right)\right\}
$$

is the action of the anisotropic four-dimensional gaussian model [9]. The couplings

$$
K_{\sigma}=\xi^{-1} k, \quad K_{\beta}=\xi k
$$

become equal on the isotropic lattice with $\xi=1$, yielding in eq. (2.11) the more familiar form of a four-dimensional "spin"-system, with a gaussian distribution for the length $\left|\tilde{\varphi}\left(x_{\alpha}\right)\right|$ of the spin attached to the site $x_{\alpha}$. For $\xi=1$ the critical case $m=0$ corresponds to the critical coupling $\frac{1}{4}$ in the gaussian model.

To evaluate the partition function, eq. (2.9), further, we go over to the reciprocal lattice of momentum coordinates and introduce Fourier-transformed field variables

$$
\begin{aligned}
\varphi_{q} & =\frac{1}{\sqrt{N_{\sigma}^{3} N_{\beta}}} \sum_{\alpha} \mathrm{e}^{-i q x_{\alpha}} \tilde{\varphi}\left(x_{\alpha}\right), \\
\tilde{\varphi}\left(x_{\alpha}\right) & =\frac{1}{\sqrt{N_{\sigma}^{3} N_{\beta}}} \sum_{q} \mathrm{e}^{i q x_{\alpha}} \varphi_{q},
\end{aligned}
$$

where the momenta $q$ have the following values in the first Brillouin zone of the reciprocal lattice:

$$
\begin{array}{r}
\mu=0: q_{0}=\frac{2 \pi}{N_{\beta} a_{\beta}} j_{0}, \quad j_{0}=0, \pm 1, \ldots \pm\left(\frac{1}{2} N_{\beta}-1\right), \frac{1}{2} N_{\beta} ; \\
\mu=1,2,3: q_{\mu}=\frac{2 \pi}{N_{\sigma} a_{\sigma}} j_{\mu}, \quad j_{\mu}=0, \pm 1, \ldots \pm\left(\frac{1}{2} N_{\sigma}-1\right), \frac{1}{2} N_{\sigma} .
\end{array}
$$

Here we have for simplicity assumed $N_{\beta}$ and $N_{\sigma}$ to be even. With the above transformation and the completeness relation

$$
\sum_{\alpha} \mathrm{e}^{i q x_{\alpha}}=N_{\sigma}^{3} N_{\beta} \delta_{q, 0},
$$

one gets for the action

$$
S=\frac{1}{2} k \xi^{-1} \sum_{q} \varphi_{q} \varphi_{q}^{*} G^{-1}\left(a_{\sigma}, \xi, q\right),
$$

where we have used the fact that the new variables $\varphi_{q}$ fulfill the condition

$$
\varphi_{-q}=\varphi_{q}^{*},
$$

since the original site variables $\tilde{\varphi}\left(x_{\alpha}\right)$ are real. The dimensionless lattice propagator $G\left(a_{\sigma}, \xi, q\right)$ is given by

$$
G^{-1}\left(a_{\sigma}, \xi, q\right)=\left(m a_{\sigma}\right)^{2}+4 \sum_{\mu=1}^{3} \sin ^{2}\left(\frac{1}{2} q_{\mu} a_{\sigma}\right)+4 \xi^{2} \sin ^{2}\left(\frac{1}{2} q_{0} a_{\beta}\right) .
$$


Due to eq. (2.17) the complex variables $\varphi_{q}$ are not all independent and it suffices to sum only over half of the reciprocal lattice. The integration measure then becomes [10]

$$
\prod_{\alpha} \mathrm{d} \tilde{\varphi}\left(x_{\alpha}\right)=2^{N_{\sigma}^{3} N_{\beta} / 2} \prod_{q \geqslant 0} \mathrm{~d} \varphi_{q} .
$$

Thus we have reduced the partition function for a free Bose field theory on a euclidean lattice to a product of gaussian integrals, and we therefore finally obtain

$$
Z_{\mathrm{E}}\left(N_{\sigma}, N_{\beta}, a_{\sigma}, \xi\right)=\xi^{N_{\sigma}^{3} N_{\beta}} \prod_{q} G^{1 / 2}\left(a_{\sigma}, \xi, q\right) .
$$

From this expression we find for the unnormalized free energy density

$$
\beta f_{\mathrm{E}}=-\frac{1}{N_{\sigma}^{3} a_{\sigma}^{3}} \ln Z_{\mathrm{E}}=-\frac{N_{\beta}}{a_{\sigma}^{3}} \ln \xi+\frac{1}{2 N_{\sigma}^{3} a_{\sigma}^{3}} \sum_{q} \ln G^{-1}\left(a_{\sigma}, \xi, q\right) .
$$

The physical free energy density $f$ is obtained from this expression by subtracting the vacuum contribution

$$
f_{\mathrm{v}}=\lim _{N_{\mathrm{B}} \rightarrow \infty} f_{\mathrm{E}},
$$

i.e. we normalize the ground-state energy of our hamiltonian to zero. Explicitly we get in the limit $N_{\beta} \rightarrow \infty$

$$
f_{\mathrm{v}} a_{\sigma}^{4}=\xi \ln (2 / \xi)+\frac{\xi}{2 N_{\sigma}^{3}} \sum_{j} \int_{-1 / 2}^{1 / 2} \mathrm{~d} x \ln \left(b^{2}(j)+\xi^{2} \sin ^{2}(\pi x)\right)
$$

with

$$
b^{2}(j)=\left(\frac{1}{2} m a_{\sigma}\right)^{2}+\sum_{\mu=1}^{3} \sin ^{2}\left(\pi j_{\mu} / N_{\sigma}\right) .
$$

The integral in eq. (2.23) is known and we finally have

$$
f_{\mathrm{v}} a_{\sigma}^{4}=-\xi \ln \xi+\frac{\xi}{N_{\sigma}^{3}} \sum_{j} \ln \left(b+\sqrt{\xi^{2}+b^{2}}\right) .
$$

Combining eqs. (2.21) and (2.25) yields the physical free energy density

$$
f a_{\sigma}^{4}=\left(f_{\mathrm{E}}-f_{\mathrm{v}}\right) a_{\sigma}^{4} .
$$

For the $m=0$ case, we have to suppress the $q=0$ term in the summation (2.21), since on a finite lattice with periodic boundary conditions it would lead to a logarithmic divergence. In the limit $N_{\sigma} \rightarrow \infty$, when the sum in (2.21) becomes an integral, this divergence disappears. On a finite lattice, dropping $q=0$ is equivalent to the suppression of the integration over a constant field configuration in the partition function (2.5). 
Using eq. (2.26) we can now calculate all other physical quantities on the lattice. In the continuum the energy density $\varepsilon$ and the pressure $p$ are given by

$$
\begin{aligned}
& \varepsilon=\frac{\partial}{\partial \beta}(\beta f)_{V}, \\
& p=\frac{\partial}{\partial V}(-V f)_{\beta} .
\end{aligned}
$$

On the lattice we rewrite the derivatives with respect to volume $V$ and inverse temperature $\beta$ in terms of the lattice parameters $a_{\sigma}$ and $\xi$ at fixed $N_{\sigma}, N_{\beta}$ :

$$
\begin{aligned}
& \left.\frac{\partial}{\partial \beta}\right|_{V}=-\left.\frac{\xi^{2}}{N_{\beta} a_{\sigma}} \frac{\partial}{\partial \xi}\right|_{a_{\sigma}}, \\
& \left.\frac{\partial}{\partial V}\right|_{\beta}=\left.\frac{1}{3 N_{\sigma}^{3} a_{\sigma}^{2}} \frac{\partial}{\partial a_{\sigma}}\right|_{a_{\beta}}=\frac{1}{3 N_{\sigma}^{3} a_{\sigma}^{2}}\left(\left.\frac{\partial}{\partial a_{\sigma}}\right|_{\xi}+\left.\frac{\xi}{a_{\sigma}} \frac{\partial}{\partial \xi}\right|_{a_{\sigma}}\right) .
\end{aligned}
$$

From eq. (2.26) and eq. (2.27a) one obtains then the energy density on a finite euclidean lattice

$$
\varepsilon a_{\sigma}^{4}=-\frac{\xi^{3}}{N_{\sigma}^{3} N_{\beta}} \sum_{j}^{\prime} \frac{\sin ^{2}\left(\pi j_{0} / N_{\beta}\right)}{b^{2}+\xi^{2} \sin ^{2}\left(\pi j_{0} / N_{\beta}\right)}+\frac{\xi^{3}}{N_{\sigma}^{3}} \sum_{j}\left(b \sqrt{\xi^{2}+b^{2}}+\xi^{2}+b^{2}\right)^{-1} .
$$

Here $\Sigma^{\prime}$ indicates that for $m=0$ the $j=0$ term does not contribute to the sum.

For a massless Bose gas the well-known relation between energy density and pressure,

$$
p=\frac{1}{3} \varepsilon,
$$

holds even on a finite lattice.

We now want to study a little more in detail the behaviour of the energy density on finite euclidean lattices, to see how well the continuum Stefan-Boltzmann law

$$
\varepsilon_{\mathrm{SB}}=\frac{1}{30} \pi^{2} \beta^{-4}
$$

is approximated.

First of all, we note that the euclidean formulation contains two different approximations to the continuum result. One consists in replacing the space continuum by a finite lattice $\left(N_{\sigma}<\infty\right)$, the second in approximating the hamiltonian partition function on a finite three-dimensional lattice by making, in addition, the temperature direction discrete $\left(N_{\beta}<\infty\right)$.

Let us check first how well the hamiltonian energy density on a finite lattice,

$$
\varepsilon_{\text {disc }} a_{\sigma}^{4}=\frac{2 \pi}{N_{\sigma}^{4}} \sum_{j} \frac{w \exp \left\{-2 \pi \beta w / N_{\sigma} a_{\sigma}\right\}}{1-\exp \left\{-2 \pi \beta w / N_{\sigma} a_{\sigma}\right\}},
$$




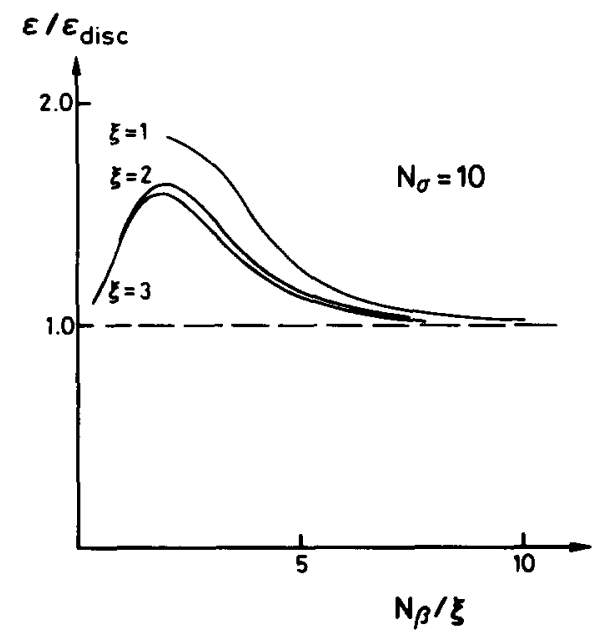

Fig. 1. The ratio between the discrete euclidean $(\varepsilon)$ and discrete hamiltonian $\left(\varepsilon_{\text {disc }}\right)$ version of the energy density of a free massless Bose gas versus inverse temperature $1 /\left(a_{\sigma} T\right)=N_{\beta} / \xi$, for $N_{\sigma}=10$ and various $\xi$.

with

$$
w=\left(\sum_{\mu=1}^{3} j_{\mu}^{2}\right)^{1 / 2},
$$

is approximated by the euclidean version eq. (2.29). In fig. 1 we show the ratio $\varepsilon / \varepsilon_{\text {disc }}$ in the case of massless bosons for $N_{\sigma}=10$ and various $\xi$ as function of $1 /\left(T a_{\sigma}\right)=N_{\beta} / \xi$. The limit $\xi \rightarrow \infty$ at fixed $N_{\beta} / \xi$ yields the hamiltonian form at fixed finite temperature, that for $N_{\beta} \rightarrow \infty$ at fixed $\xi$ the hamiltonian form at $T=0$.

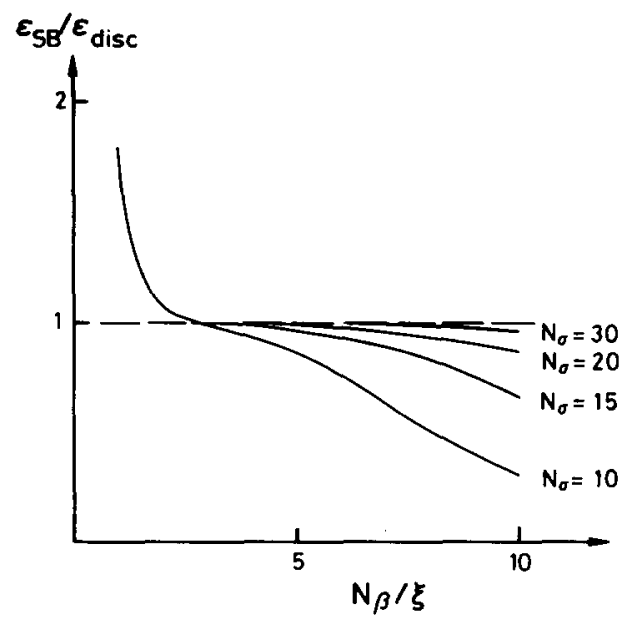

Fig. 2. The ratio between the continuum $\left(\varepsilon_{\mathrm{SB}}\right)$ and discrete hamiltonian $\left(\varepsilon_{\mathrm{disc}}\right)$ version of the energy density of a free massless Bose gas versus inverse temperature $1 /\left(a_{\sigma} T\right)=N_{\beta} / \xi$, for various $N_{\sigma}$. 


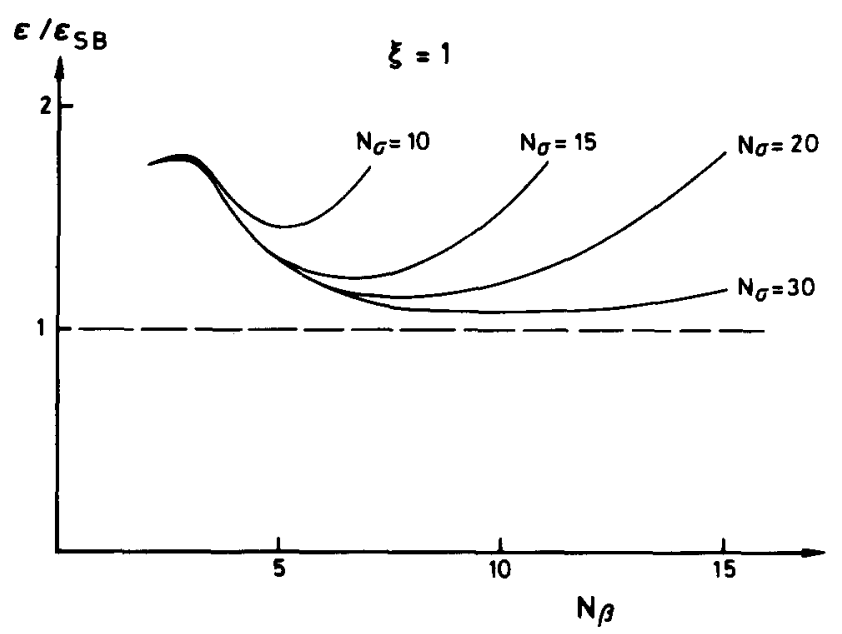

Fig. 3. The ratio $\varepsilon / \varepsilon_{\mathrm{SB}}$ versus $N_{\beta}$ for $\xi=1$ and various $N_{\sigma}$.

The discrete hamiltonian version is itself only an approximation to the continuum form $\varepsilon_{\mathrm{SB}}$. In fig. 2 we note that $\varepsilon_{\mathrm{SB}} / \varepsilon_{\mathrm{disc}}$ approaches unity for $N_{\sigma} \rightarrow \infty$ at fixed $1 /\left(a_{\sigma} T\right)=N_{\beta} / \xi$; for $N_{\beta} \rightarrow \infty$ at fixed $\xi, a_{\sigma}$ and $N_{\sigma}$, i.e., in the low temperature limit, the approximation becomes arbitrarily bad. The reason for this is that on a finite spatial lattice, we lose the low momenta $\left(\leqslant 1 /\left(N_{\sigma} a_{\sigma}\right)\right)$, and at low temperatures, these give the dominant contributions to $\varepsilon_{\mathrm{SB}}$.

If we compare the $\xi=1, N_{\sigma}=10$ curves in figs. 1 and 2 , we see that an increase of $N_{\beta}$ improves the approximation of $\varepsilon / \varepsilon_{\text {disc }}$, while it makes that of $\varepsilon_{\mathrm{SB}} / \varepsilon_{\text {disc }}$ worse. Combining these opposing effects, we obtain for $\varepsilon / \varepsilon_{\mathrm{SB}}$ the behaviour shown in fig. 3. Corresponding results for other $N_{\sigma}$ are also included, while those for different $\xi$ are shown in fig. 4.

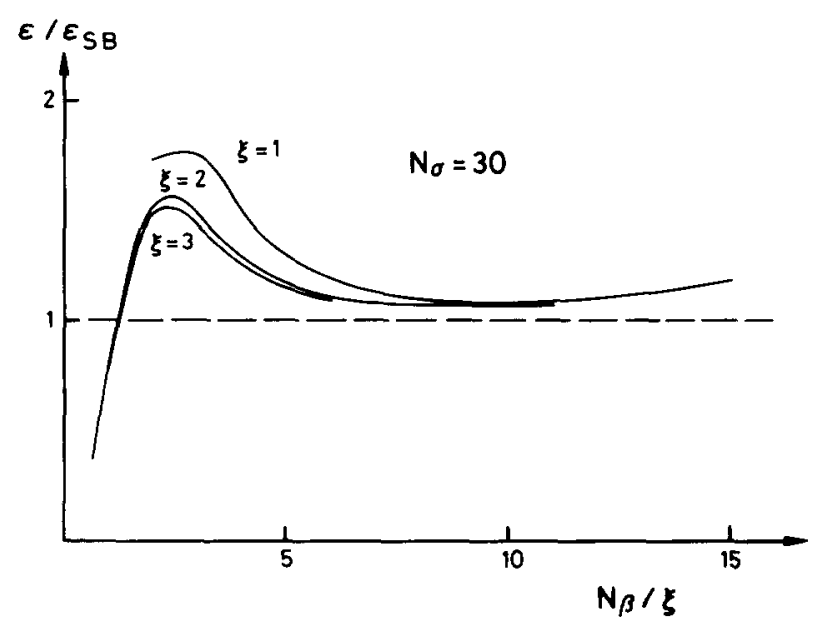

Fig. 4. The ratio $\varepsilon / \varepsilon_{\mathrm{SB}}$ versus $N_{\beta} / \xi$ for $N_{\sigma}=30$ and various $\xi$. 
From the behaviour of the energy density we conclude that the influence of lattice size for finite temperature calculations on small lattices is by no means small. Qualitatively we note:

too large or too small a value of $N_{\beta}$ at fixed $\xi$ and $N_{\sigma}$ make the lattice approximation worse;

increasing $N_{\sigma}$ at fixed $\xi$ and large $N_{\beta}$ improves the lattice approximation;

large $\xi$ at fixed $N_{\beta}$ and $N_{\sigma}$ make the lattice approximation worse.

In the Monte Carlo simulation of more complicated systems, like SU(2) gauge models, one can, in general, not do the subtraction of the vacuum contribution by just taking the limit $N_{\beta} \rightarrow \infty$. In practice, one may then approximate physical quantities by taking $N_{\beta}=N_{\sigma}$ as the $T=0$ contribution and keeping $N_{\beta} \ll N_{\sigma}$ for the remaining finite temperature term. In table 1 we present, therefore, the ratio $\varepsilon / \varepsilon_{\mathrm{SB}}$ for some small lattices with $\xi=1$, for both these definitions of the vacuum term $\varepsilon_{V}$. They are seen to give quite compatible results, if $N_{\beta}$ is indeed much smaller than $N_{\sigma}$.

The high temperature limit of $\mathrm{SU}(N)$ gauge theories is also expected to be of Stefan-Boltzmann form, as confirmed by a weak coupling expansion on the lattice $[10]^{\star}$. The Monte Carlo evaluation for SU(2) $[1,11]$ and $S U(3)[12]$ leads to ratios $\varepsilon / \varepsilon_{\mathrm{SB}}$ which at high $T$ within errors agree with the values of table 1 .

\section{The ideal Fermi gas on the euclidean lattice}

In the previous section we have discussed in detail how one can calculate thermodynamic quantities on a finite euclidean lattice. In this section we shall therefore mention only the new features coming in, when one tries to deal with fermions on a lattice.

TABLE 1

The ratio $\varepsilon / \varepsilon_{\mathrm{SB}}=\left(\varepsilon_{\mathrm{E}}-\varepsilon_{\mathrm{v}}\right) / \varepsilon_{\mathrm{SB}}$ with the vacuum energy defined as (a) $\varepsilon_{\mathrm{v}}=\lim _{N_{\beta} \rightarrow \infty} \varepsilon_{\mathrm{E}}$ or (b) as the value of $\varepsilon_{\mathrm{E}}$ on a symmetric lattice $N_{\sigma}=N_{\beta}$

\begin{tabular}{|c|c|c|c|c|c|c|c|c|}
\hline$N_{\sigma}$ & \multicolumn{2}{|c|}{7} & \multicolumn{2}{|c|}{8} & \multicolumn{2}{|c|}{9} & \multicolumn{2}{|c|}{10} \\
\hline & a & b & a & b & $\mathbf{a}$ & b & $\mathbf{a}$ & b \\
\hline 2 & 1.7543 & 1.7314 & 1.7468 & 1.7336 & 1.7429 & 1.7347 & 1.7407 & 1.7353 \\
\hline 3 & 1.8488 & 1.7329 & 1.8113 & 1.7441 & 1.7915 & 1.7498 & 1.7803 & 1.7531 \\
\hline 4 & 1.7743 & 1.4081 & 1.6587 & 1.4463 & 1.5969 & 1.4653 & 1.5617 & 1.4757 \\
\hline 5 & 1.9569 & 1.0627 & 1.6877 & 1.1691 & 1.5409 & 1.2193 & 1.4561 & 1.2461 \\
\hline 6 & 2.4993 & 0.6453 & 1.9765 & 0.9013 & 1.6852 & 1.0183 & 1.5143 & 1.0786 \\
\hline
\end{tabular}

* A slight extension of the weak coupling expansion, which in ref. [10] was performed for zero temperature, yields at finite temperature to lowest order the free gluon gas. 
In general there is no way of putting fermions on the lattice without doubling the number of species and preserving at the same time chiral invariance (for $m=0$ ) and locality of the derivative. There are several proposals how to avoid species doubling on the lattice [13], but for the actual Monte Carlo simulation of fermions in general the original prescription of Wilson [14] has been used. He introduces an explicit chiral symmetry breaking term into the action, which however disappears in the continuum limit. In this way the lattice action for free fermions (spin $\frac{1}{2}$ ) becomes

with

$$
S_{f}(\psi, \bar{\psi})=\sum_{x} \bar{\psi}(x) \psi(x)-\sum_{x, y}\left\{K_{\sigma} \sum_{\mu=1}^{3} \bar{\psi}(x) D_{x, y}^{\mu} \psi(y)+K_{\beta} \bar{\psi}(x) D_{x, y}^{0} \psi(y)\right\},
$$

$$
D_{x, y}^{\mu}=\delta_{y, x+e_{\mu}}\left(1-\gamma_{\mu}\right)+\delta_{x, y+e_{\mu}}\left(1+\gamma_{\mu}\right), \quad \mu=0,1,2,3 .
$$

Here $x$ and $y$ denote a summation over all lattice sites and an implicit summation of the Dirac indices is always understood. The $\gamma_{\mu}$ 's are the Dirac matrices and $\psi(x), \bar{\psi}(x)$ denote dimensionless spinor variables at the lattice site $x$. The couplings $K_{\sigma}$ and $K_{\beta}$ are given by

$$
K_{\sigma}=\frac{1}{2} \xi^{-1} k_{\mathrm{f}}, \quad K_{\beta}=\frac{1}{2} k_{\mathrm{f}},
$$

with

$$
k_{\mathrm{f}}^{-1}=m a_{\sigma} \xi^{-1}+3 \xi^{-1}+1 .
$$

Thus we get for the partition function of free spin $\frac{1}{2}$ fermions and antifermions on the finite euclidean lattice of size $N_{\beta} \times N_{\sigma}^{3}$

$$
Z_{\mathrm{E}}\left(N_{\sigma}, N_{\beta}, a_{\sigma}, \xi\right)=\int_{\mathrm{ap}} \prod_{x}[\mathrm{~d} \psi(x) \mathrm{d} \bar{\psi}(x)] \mathrm{e}^{-S_{\mathrm{f}}(\psi, \bar{\psi})} .
$$

Here ap denotes that we have to take antiperiodic boundary conditions for the fermionic field variables in $\beta$-direction. The integral (3.5) has to be understood as an integral over non-commuting Grassmann variables. Furthermore, we have neglected all multiplicative terms, which drop out in physical quantities after subtracting the vacuum terms.

On the reciprocal lattice the action (3.1) becomes

$$
S_{\mathrm{f}}\left(\psi_{q}, \bar{\psi}_{q}\right)=-\sum_{q} \bar{\psi}_{q} \Delta_{q}^{\mathrm{f}} \psi_{q},
$$

with

$$
\begin{aligned}
\Delta_{q}^{\mathrm{f}}= & 2 K_{\sigma} \sum_{\mu=1}^{3} \cos \left(q_{\mu} a_{\sigma}\right)+2 K_{\beta} \cos \left(q_{0} a_{\beta}\right)-1 \\
& -2 i K_{\sigma} \sum_{\mu=1}^{3} \gamma_{\mu} \sin \left(q_{\mu} a_{\sigma}\right)-2 i K_{\beta} \gamma_{o} \sin \left(q_{0} a_{\beta}\right) .
\end{aligned}
$$


For $\mu=1,2,3$ the momentum values $q_{\mu}$ are given by eq. (2.14), whereas due to the antiperiodic boundary conditions in the $\beta$-direction the zeroth component $q_{0}$ is given by

$$
q_{0}=\frac{2 \pi}{N_{\beta} a_{\beta}}\left(j_{0}+\frac{1}{2}\right) .
$$

The partition function then becomes

$$
Z_{\mathrm{E}}=\prod_{q} \operatorname{det} \Delta_{q}^{\mathrm{f}},
$$

with

$$
\begin{aligned}
\operatorname{det} \Delta_{q}^{\mathrm{f}}= & {\left[\left(1-2 K_{\beta}-6 K_{\sigma}+4 K_{\beta} \sin ^{2}\left(\frac{1}{2} q_{0} a_{\beta}\right)+4 K_{\sigma} \sum_{\mu=1}^{3} \sin ^{2}\left(\frac{1}{2} q_{\mu} a_{\sigma}\right)\right)^{2}\right.} \\
& \left.+4 K_{\beta}^{2} \sin ^{2}\left(q_{0} a_{\beta}\right)+4 K_{\sigma}^{2} \sum_{\mu=1}^{3} \sin ^{2}\left(q_{\mu} a_{\sigma}\right)\right]^{2} .
\end{aligned}
$$

To stress the similarity between eqs. (3.9), (3.10) and the corresponding result for bosons, eqs. (2.18), (2.20), we use the explicit form of the couplings $K_{\sigma}$ and $K_{\beta}$. The evaluation of the unnormalized free energy density yields then

$$
\beta f_{\mathrm{E}}=-\frac{4 N_{\beta}}{a_{\sigma}^{3}} \ln \left(k_{\mathrm{f}} / \xi\right)-\frac{2}{N_{\sigma}^{3} a_{\sigma}^{3}} \sum_{j} \ln \left[B^{2}+A+4 \xi(B+\xi) \sin ^{2}\left(\pi\left(j_{0}+\frac{1}{2}\right) / N_{\beta}\right)\right],
$$

with

$$
\begin{aligned}
& A=\sum_{\mu=1}^{3} \sin ^{2}\left(2 \pi j_{\mu} / N_{\sigma}\right), \\
& B=m a_{\sigma}+2 \sum_{\mu=1}^{3} \sin ^{2}\left(\pi j_{\mu} / N_{\sigma}\right) .
\end{aligned}
$$

Thus it becomes clear from eq. (3.11) that we can calculate the vacuum contribution to the free energy in the same way as in the bosonic case. We get

$$
f_{\mathrm{v}} a_{\sigma}^{4}=-4 \xi \ln \left(k_{\mathrm{f}} / \xi\right)-\frac{4 \xi}{N_{\sigma}^{3}} \sum_{j} \ln \left[\frac{1}{2}\left(\sqrt{B^{2}+A}+\sqrt{B^{2}+A+4 \xi(B+\xi)}\right)\right] .
$$

The physical free energy density is then again given by eq. (2.26) and, using eqs. (2.27a) and (2.28a), one can calculate the energy density of spin $\frac{1}{2}$ fermions and antifermions with free creation and annihilation (chemical potential $\mu_{\mathrm{f}} \equiv 0$ ). This yields

$$
\varepsilon a_{\sigma}^{4}=\left(\varepsilon_{\mathrm{E}}-\varepsilon_{\mathrm{v}}\right) a_{\sigma}^{4},
$$


with

$$
\begin{gathered}
\varepsilon_{\mathrm{E}} a_{\sigma}^{4}=\frac{8 \xi^{2}}{N_{\sigma}^{3} N_{\beta}} \sum_{j} \frac{(B+2 \xi) \sin ^{2}\left(\pi\left(j_{0}+\frac{1}{2}\right) / N_{\beta}\right)}{B^{2}+A+4 \xi(B+\xi) \sin ^{2}\left(\pi\left(j_{0}+\frac{1}{2}\right) / N_{\beta}\right)}-4 \xi k_{\mathrm{f}} \\
\varepsilon_{\mathrm{v}} a_{\sigma}^{4}=\frac{8 \xi^{2}}{N_{\sigma}^{3}} \sum_{j} \frac{B+2 \xi}{\left[\left(B^{2}+A\right)\left(B^{2}+A+4 \xi(B+\xi)\right)\right]^{1 / 2}+B^{2}+A+4 \xi(B+\xi)}-4 \xi k_{\mathrm{f}} .
\end{gathered}
$$

In the case of massless fermions we can again compare the above result with the Stefan-Boltzmann form of the energy density of free massless fermion-antifermion pairs in the continuum:

$$
\varepsilon_{\mathrm{f} \overline{\mathrm{f}}}=\frac{7}{60} \pi^{2} \beta^{-4} .
$$

As in the bosonic case we have calculated the ratio $\varepsilon / \varepsilon_{\overline{\mathrm{f}} \overline{\mathrm{f}}}$ for finite lattices and different values of $\xi$. In fig. 5 we show the results for $\xi=1$ and various $N_{\sigma}$ as a function of $N_{\beta}$. Corresponding results for other values of $\xi$ are shown in fig. 6 . We notice that for small values of $N_{\beta}$ the deviation from the continuum result is approximately twice as big as in the case of free bosons. In the limit $N_{\beta} / \xi \rightarrow \infty$ the ratio $\varepsilon / \varepsilon_{\mathrm{f}}$ goes to zero, whereas the corresponding quantity for bosons, $\varepsilon / \varepsilon_{\mathrm{SB}}$, diverges in this limit. The reason for this is the different behaviour of the discrete hamiltonian versions of the energy density in both cases. As seen in fig. 2, the ratio $\varepsilon_{\mathrm{SB}} / \varepsilon_{\text {disc }}$ for bosons goes to zero in the limit $N_{\beta} / \xi \rightarrow \infty$. The corresponding ratio for fermions diverges, as shown in fig. 7. This difference is due to the different finite lattice approximation of the low momentum region in the two cases.

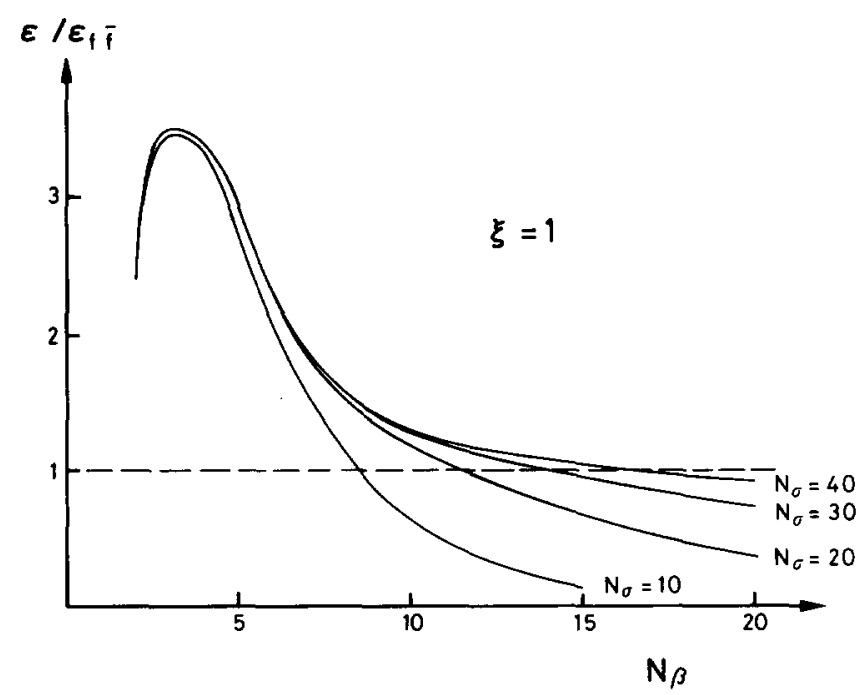

Fig. 5. The ratio between the discrete euclidean $(\varepsilon)$ and continuum $\left(\varepsilon_{\mathbf{f}}\right)$ version of the energy density of free massless fermions and antifermions versus $N_{\beta}$, for $\xi=1$ and various $N_{\sigma}$. 


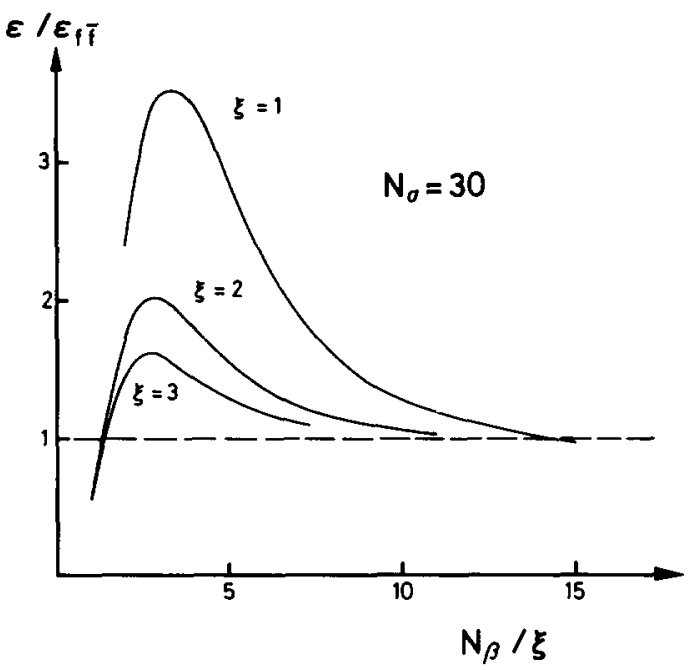

Fig. 6. The ratio $\varepsilon / \varepsilon_{\mathrm{f} \tilde{\mathrm{f}}}$ versus $N_{\beta} / \xi$ for $N_{\sigma}=30$ and various $\xi$.

\section{Conclusions}

We have calculated the behaviour of thermodynamic quantities on finite euclidean lattices for free bosons and fermions and found that the influence of finite lattice size on quantities such as the energy density is by no means small. Therefore, we also expect in the case of more complicated interacting systems, like $\mathrm{SU}(N)$ gauge theories, that one cannot obtain the correct finite temperature continuum values by Monte Carlo simulation on small lattices. One can, however, use the results for

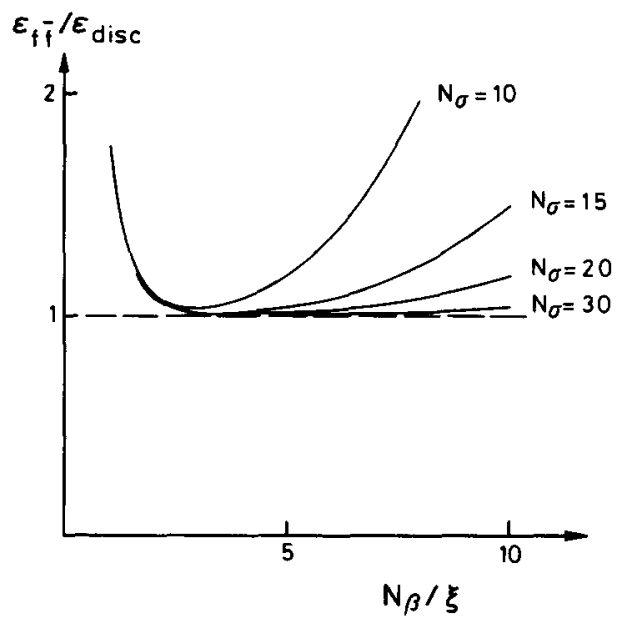

Fig. 7. The ratio between the continuum $\left(\varepsilon_{\mathrm{ff}}\right)$ and discrete hamiltonian $\left(\varepsilon_{\text {disc }}\right)$ version of the energy density of a free massless Fermi gas versus inverse temperature $1 /\left(a_{\sigma} T\right)=N_{\beta} / \xi$, for various $N_{\sigma}$. 
the ideal gases on finite lattices as a reference measure and study the finite size dependence of the interacting theory in comparison to this reference. Such a procedure was shown to be quite successful in the case of pure SU(2) and SU(3) gauge theories $[11,12]$ at high temperatures, where a correction using the coefficients of table 1 was found to remove discrepancies between calculations on different size lattices. Finally, we note again that the lattice approximation for free Bose and Fermi fields at finite temperature becomes optimal for specific lattices (large $N_{\beta}$ with $N_{\beta} \ll N_{\sigma}$ ). Hence we expect increasing finite size deviations also for $\mathrm{SU}(N)$ gauge field simulations if we leave this region of optimal approximation.

We thank I. Montvay for useful discussions.

\section{References}

[1] J. Engels, F. Karsch, I. Montvay and H. Satz, Phys. Lett. 101B (1981) 89

[2] L.D. McLerran and B. Svetitsky, Phys. Lett. 98B (1981) 195

[3] J. Kuti, J. Polónyi and K. Szlachányi, Phys. Lett. 98B (1981) 199

[4] J. Engels, F. Karsch, I. Montvay and H. Satz, Phys. Lett. 102B (1981) 332

[5] M. Creutz, Phys. Rev. D21 (1980) 2308

[6] M. Nauenberg, T. Schalk and R. Brower, Phys. Rev. D24 (1981) 548

[7] C.B. Lang and H., Nicolai, Nucl. Phys. B200 [FS4] (1982) 135

[8] C. Bernard, Phys. Rev. D9 (1974) 3312

[9] T.H. Berlin and M.Kac, Phys. Rev. 86 (1952) 821

[10] V.F. Müller and W. Rühl, Ann. of Phys. 133 (1981) 240

[11] J. Engels, F. Karsch, I. Montvay and H. Satz, Gauge field thermodynamics for the SU(2) Yang-Mills system, Bielefeld preprint BI-TP 81/29 (December 1981)

[12] I. Montvay and E. Pietarinen, Stefan-Boltzmann law at high temperature for the gluon gas, DESY preprint 81-077 (December 1981)

[13] P. Becher, Proc. Padova-Würzburg Symp. on Problems in gauge theories, ed. M. Böhm, p. 65; S. Elitzur, Int. Conf. on High-energy physics, ed. J. Dias de Deus, Lisbon, 1981

[14] K.G. Wilson, Phys. Rev. D10 (1974) 2445 